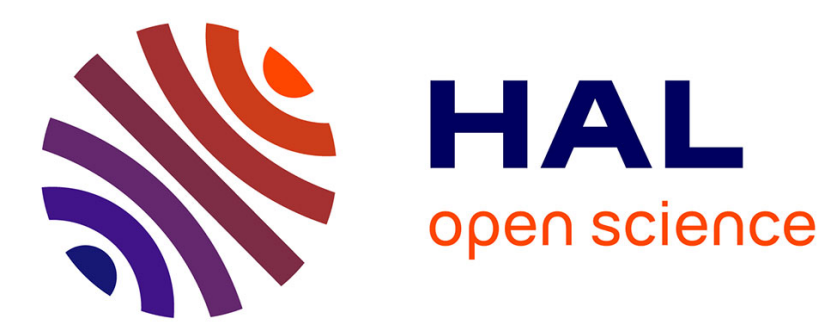

\title{
Global linear stability of the non-parallel Batchelor vortex
}

\author{
C.J. Heaton, J.W. Nichols, Peter J. Schmid
}

\section{To cite this version:}

C.J. Heaton, J.W. Nichols, Peter J. Schmid. Global linear stability of the non-parallel Batchelor vortex. Journal of Fluid Mechanics, 2009, 629 (June), pp.139-160. 10.1017/s0022112009006399 . hal-01021155

\section{HAL Id: hal-01021155 \\ https://hal-polytechnique.archives-ouvertes.fr/hal-01021155}

Submitted on 15 Jul 2014

HAL is a multi-disciplinary open access archive for the deposit and dissemination of scientific research documents, whether they are published or not. The documents may come from teaching and research institutions in France or abroad, or from public or private research centers.
L'archive ouverte pluridisciplinaire HAL, est destinée au dépôt et à la diffusion de documents scientifiques de niveau recherche, publiés ou non, émanant des établissements d'enseignement et de recherche français ou étrangers, des laboratoires publics ou privés. 


\title{
Global linear stability of the non-parallel Batchelor vortex
}

\author{
C. J. HEATON†, J. W. NICHOLS AND P. J. SCHMID \\ Laboratoire d'Hydrodynamique (LadHyX), CNRS-École Polytechnique, 91128 Palaiseau, France
}

(Received 14 October 2008 and in revised form 29 January 2009)

Linear stability of the non-parallel Batchelor vortex is studied using global modes. This family of swirling wakes and jets has been extensively studied under the parallel-flow approximation, and in this paper we extend to more realistic non-parallel base flows. Our base flow is obtained as an exact steady solution of the Navier-Stokes equations by direct numerical simulation (with imposed axisymmetry to damp all instabilities). Global stability modes are computed by numerical simulation of the linearized equations, using the implicitly restarted Arnoldi method, and we discuss fully the numerical and convergence issues encountered. Emphasis is placed on exploring the general structure of the global spectrum, and in particular the correspondence between global modes and local absolute modes which is anticipated by weakly non-parallel asymptotic theory. We believe that our computed global modes for a weakly nonparallel vortex are the first to display this correspondence with local absolute modes. Superpositions of global modes are also studied, allowing an investigation of the amplifier dynamics of this unstable flow. For an illustrative case we find global nonmodal transient growth via a convective mechanism. Generally amplifier dynamics, via convective growth, are prevalent over short time intervals, and resonator dynamics, via global mode growth, become prevalent at later times.

\section{Introduction}

The concept of global stability is necessary to describe the stability properties of open shear flows such as wakes, jets and boundary layers, because of the universal feature that such flows are spatially developing. In contrast, the familiar local approach is to approximate the flow as parallel and non-developing. The detailed relationship between local and global stability is an area of active interest, e.g. see the reviews by Huerre \& Monkewitz (1990) and Chomaz (2005), particularly because modern computing is now becoming capable of the more demanding calculations required by a global analysis.

In this paper we study the global stability of the Batchelor vortex: a swirling wake/jet derived by Batchelor (1964) to model an aeronautical trailing line vortex. The flow also finds application as a good model for swirling flows in other contexts, in particular the approach and wake flows of vortex breakdown states, see Leibovich (1978) and Ruith et al. (2003). There exists an extensive body of work concerning the local stability of the Batchelor vortex under the approximation of a parallel base flow (see below for a review). It is important to relax this approximation, and hence perform a global analysis of the developing flow, both to re-assess the meaning of 
the local analyses and to improve the accuracy of the model as a representation of reality.

The Batchelor vortex also serves as a convenient prototype for global stability analysis of flows which are weakly non-parallel, i.e. with slow spatial development. For this class of flows asymptotic theory has been developed to extend local stability analysis into the global setting (Huerre \& Monkewitz 1990; Huerre \& Rossi 1998). For flows which are strictly (asymptotically) weakly non-parallel, global stability computations are still in their infancy, with only a small number of analyses performed for the flat-plate boundary layer (see below for a review). Although widely accepted, aspects of the weakly non-parallel theory have yet to be observed in practice using the full Navier-Stokes equations, and so this forms another motivation for the present work.

\subsection{Review of Batchelor vortex stability}

Local stability analysis, in the parallel-flow approximation, of the Batchelor vortex has been comprehensively studied. In terms of classical instability modes, there are centrifugal modes of inviscid type which are damped by viscosity (Lessen, Singh \& Paillet 1974; Leibovich \& Stewartson 1983; Heaton 2007a). These were the first instabilities to be discovered and are the strongest growing. There are also viscous modes which are destabilized by viscosity, analogously to Tollmien-Schlichting waves (Khorrami 1991; Fabre \& Jacquin 2004). These modes are somewhat weaker, but are present in vortices of all swirl strengths (Le Dizès \& Fabre 2007), while the inviscid modes are unstable only at small swirl (Stewartson \& Brown 1985; Heaton 2007a).

The absolute or convective character of the instability modes has been studied by Olendraru et al. (1999) for an inviscid vortex, and by Delbende, Chomaz \& Huerre (1998), Yin, Sun \& Wei (2000) and Olendraru \& Sellier (2002) at finite Reynolds number. Absolute instability is found for both jet and wake type flows with axial counter-flow, and also in wake flows without counter-flow for which the wake is sufficiently 'deep' (meaning an axial velocity defect at least $80 \%$ of the free stream). For less deep wakes and for co-flowing jets, the instabilities are purely convective in nature.

Finally, the optimal growth of linear perturbations has also been studied. Strong non-modal growth is found, in line with other canonical shear flows (Heaton \& Peake 2007). This could signal a bypass route to breakdown of the vortex, or could combine with and amplify the classical instabilities (Abid 2008). The optimal growth methodology is also found to be useful for assessing the competition of the many coexisting instability mechanisms (Heaton 2007b).

Note that in this paper we consider stability problems based on a linearized NavierStokes (LNS) approach, and thus parabolized stability equations (PSE) results are outside the scope of the present paper. See Herbert (1997) for a review of PSE, which is an approximation valid for convectively unstable flows, and see Broadhurst (2006) for some PSE results on the Batchelor vortex under the parallel-flow approximation.

Beyond the local parallel-flow approximation, we are not aware of any stability study of a spatially developing vortex. The works by Abid \& Brachet (1998) and Abid (2008) consider the stability of a parallel-flow which evolves in time to spread out laterally by viscous diffusion. This approach is an improvement upon the simplest model because the diffusing parallel vortex is an exact solution of the Navier-Stokes equations, and is convenient computationally as it retains the property of parallel flow. It is still not fully realistic, however: the most physically relevant flow should be steady in time, spatially developing and non-parallel. 
In this paper, therefore, we consider base flows which are exact steady solutions of the Navier-Stokes equations, computed by direct numerical simulation (DNS). As such, our base flow is the exact flow to which Batchelor's (1964) solution is asymptotic. With appropriate choice of parameters, we obtain a flow which is indeed nearly parallel, and which recovers Batchelor's (1964) solution as $x \rightarrow \infty$.

\subsection{Review of global stability results}

Local and global stability concepts are covered in detail by the reviews of Huerre \& Monkewitz (1990), Huerre \& Rossi (1998), Huerre (2000) and Chomaz (2005). The concepts of absolute/convective stability (in relation to parallel base flows) and global stability (in relation to non-parallel base flows) are well established. Analysis and computation are generally simpler in the local approach, but of course the global approach is better for accurately modelling real non-parallel flows. Consequently, much work has been focused on understanding the subtle but strong links which exist between the two (see the cited reviews for more details).

Thanks to advances in computational power, global stability computations are now becoming feasible to perform. For example, Pier $(2002,2008)$ and others have looked at the wake flow behind a cylinder and other bluff bodies. A kervik et al. (2007) and Marquet et al. (2008) and Marquet et al. (to appear) consider flows in curved channels with recirculation bubbles. Blackburn, Barkley \& Sherwin (2008) consider flow over a backward-facing step and Griffith et al. (2008) consider flow in stenotic arteries, which similarly have recirculation bubbles. A common feature of these flows is that they are not in a strict sense nearly parallel or slowly developing in space: the geometry causes a localized recirculating region in which the flow is far from parallel and this in turn tends to dominate the global stability results.

Existing theory assumes a flow which is asymptotically nearly parallel and slowly developing in space. Current understanding is mainly based on this theory, and on studies of model equations such as the Ginzburg-Landau equation (e.g. Cossu \& Chomaz 1997). The only works we are aware of which study base flows within the scope of the asymptotic theory are those of Ehrenstein \& Gallaire (2005) and Åkervik et al. (2008), who consider flat-plate boundary-layer flow. Computed examples of the theory applying in practice are valuable for the experience and understanding they provide, which are especially useful when moving on to more complicated flows, such as the ones cited above, to which the theory does not directly apply. As mentioned above, some aspects of the theory are still to be observed in practice, and this therefore adds another interpretation to our results as we build a picture of the global stability of the Batchelor vortex. In particular, we give fully converged global modes which correspond to local absolute instability modes in the manner predicted by theory. The global modes found by Ehrenstein \& Gallaire (2005) and Åkervik et al. (2008), whilst certainly sufficient for the superposition procedure for which those authors employ them, do not appear to be sufficiently converged to display this correspondence with local theory.

The remainder of this paper is set out as follows. In $\S 2$ the governing equations are given, and we outline our numerical methodology. In $\S 3$ we investigate the global stability of an artificial parallel base flow with boundary conditions. This is used as a means to introduce and discuss global stability concepts, and to give further details of numerical and convergence issues. In $\S 4$ the global stability of a non-parallel base flow is addressed in full. Finally, conclusions are drawn in $\S 5$. 


\section{Governing equations and solution method}

\subsection{Governing equations}

We consider incompressible flow of constant density satisfying the Navier-Stokes equations. All quantities are made non-dimensional using the constant fluid density, the nominal vortex core radius and the velocity of the fluid in the free stream. We use cylindrical coordinates $(x, r, \theta)$ aligned along the axis of the vortex core.

A steady base flow is assumed in the form

$$
\boldsymbol{U}=U(x, r) \boldsymbol{e}_{x}+V(x, r) \boldsymbol{e}_{r}+W(x, r) \boldsymbol{e}_{\theta},
$$

which satisfies the full Navier-Stokes equations:

$$
\left.\begin{array}{rl}
\frac{\partial \boldsymbol{U}}{\partial t}+\boldsymbol{U} \cdot \nabla \boldsymbol{U} & =-\nabla P+\frac{1}{R e} \nabla^{2} \boldsymbol{U}, \\
\nabla \cdot \boldsymbol{U} & =0 .
\end{array}\right\}
$$

According to Batchelor (1964), a steady solution of (2.2) is given asymptotically as $x \rightarrow \infty$ by

$$
\begin{aligned}
U & \sim 1-\alpha(x) \mathrm{e}^{-r^{2} / \delta(x)^{2}}, \\
V & \sim 0 \\
W & \sim \frac{\kappa}{r}\left(1-\mathrm{e}^{-r^{2} / \delta(x)^{2}}\right),
\end{aligned}
$$

where

$$
\begin{aligned}
\kappa & =\text { constant } \\
\alpha(x) & =\kappa^{2} \frac{\operatorname{Re} \log (x / R e)}{8 x}, \\
\delta(x) & =2 \sqrt{x / \operatorname{Re}} .
\end{aligned}
$$

The quantity $\delta(x)$ corresponds physically to the local vortex core radius, while $\alpha(x)$ is the axial velocity defect and $\kappa$ is a swirl strength parameter.

A simple parallel-flow approximation to the Batchelor vortex can be obtained by replacing ' $\sim$ ' with ' $=$ ' in (2.3)-(2.5) and approximating $\alpha$ and $\delta$ as constants. The resulting flow is variously referred to as the 'Batchelor vortex' or the 'q-vortex', and forms the basis for the local stability analyses reviewed in $\S 1.1$. The results of those studies are usually presented in terms of $q$, a dimensionless measure of the vortex swirl strength which is given in terms of the present variables by

$$
q=\kappa /(\alpha \delta) \text {. }
$$

In the present paper, we shall instead derive a steady base flow by directly solving (2.2) numerically. Working with a correct base flow which is a solution of (2.2) is an important improvement, and it is this which necessitates the global stability analysis we undertake.

The LNS equations governing small perturbations in velocity $\boldsymbol{u}$ and pressure $p$ to a steady base flow $\boldsymbol{U}(x, r)$ are given by

$$
\left.\begin{array}{rl}
\frac{\partial \boldsymbol{u}}{\partial t}+\boldsymbol{u} \cdot \nabla \boldsymbol{U}+\boldsymbol{U} \cdot \nabla \boldsymbol{u} & =-\nabla p+\frac{1}{R e} \nabla^{2} \boldsymbol{u} \\
\nabla \cdot \boldsymbol{u} & =0 .
\end{array}\right\}
$$


Since the base flow $\boldsymbol{U}(x, r)$ is independent of time $t$ and polar angle $\theta$, we may assume the perturbations to be in the form

$$
\left.\begin{array}{l}
\boldsymbol{u}=\boldsymbol{u}(x, r) \mathrm{e}^{\mathrm{i} m \theta-\mathrm{i} \omega t}, \\
p=p(x, r) \mathrm{e}^{\mathrm{i} m \theta-\mathrm{i} \omega t},
\end{array}\right\}
$$

where $m \in \mathbb{Z}$ is the azimuthal wavenumber and $\omega \in \mathbb{C}$ is the frequency. Inserting (2.11) into (2.10) gives

$$
\boldsymbol{A} \boldsymbol{q}=\mathrm{i} \omega \boldsymbol{B} \boldsymbol{q}
$$

In (2.12) we have $\boldsymbol{q}=(u, v, w, p)^{\mathrm{t}}$, the operators $\boldsymbol{A}$ and $\boldsymbol{B}$ are given by

$$
\begin{aligned}
& \boldsymbol{A}=\left(\begin{array}{cccc}
C+U_{x}-D & U_{r} & 0 & \partial_{x} \\
V_{x} & C+V_{r}-\widetilde{D} & -2 W / r+R e^{-1} 2 \mathrm{i} m / r^{2} & \partial_{r} \\
W_{x} & W_{r}+W / r-R e^{-1} 2 \mathrm{i} m / r^{2} & C+V / r-\widetilde{D} & \mathrm{i} m / r \\
\partial_{x} & r^{-1} \partial_{r} r & \mathrm{i} m / r & 0
\end{array}\right), \\
& \boldsymbol{B}=\left(\begin{array}{llll}
1 & 0 & 0 & 0 \\
0 & 1 & 0 & 0 \\
0 & 0 & 1 & 0 \\
0 & 0 & 0 & 0
\end{array}\right) \\
& \left.\begin{array}{rl}
C & =U \partial_{x}+V \partial_{r}+\mathrm{i} m W / r \\
D & =R e^{-1}\left(\partial_{x}^{2}+r^{-1} \partial_{r} r \partial_{r}-m^{2} / r^{2}\right) \\
\widetilde{D} & =D-R e^{-1} r^{-2}
\end{array}\right\}
\end{aligned}
$$

and subscripts denote partial differentiation. On specifying appropriate boundary conditions, details of which are given below, (2.12) is an eigenvalue problem to solve for $\omega$. The eigenfunctions are two-dimensional, having dependence on $x$ and $r$, and it is this which makes the computation significantly more challenging than in the local framework.

\subsection{Computational method}

Our DNS are based on the code developed by Nichols (2005) for flows in cylindrical geometry. Sixth-order compact finite difference schemes are used to compute spatial derivatives in the axial and radial directions, and a spectral representation is used for the angular coordinate. For time stepping we use a fourth-order Runge-Kutta scheme, coupled with a pressure projection method to treat correctly the incompressibility condition. This projection requires solving a Poisson problem for the pressure at each time step. The difficulties arising from the coordinate singularity at $r=0$ in cylindrical coordinates are treated by the asymptotic method derived by Constantinescu \& Lele (2002). See Nichols (2005), and also Nichols, Schmid \& Riley (2007) and Nichols \& Schmid (2008) for further details of the DNS code. The fully nonlinear DNS is used to obtain base flows, whereas a linearized version of the code is used for stability analysis.

\subsection{Obtaining the steady base flow}

We obtain a steady base flow in the form of a steady solution to (2.2). A computational box is defined, typically $0 \leqslant r \leqslant 10$ and $0 \leqslant x \leqslant 250$, and the equations are integrated forwards in time. On the inlet boundary $(x=0)$ we impose flow profiles corresponding to Batchelor's vortex solution, taking (2.3)-(2.5) for chosen values of $\alpha(0), \kappa(0)$ and $\delta(0)$. On the lateral boundaries a traction-free boundary condition is applied (see 
Nichols et al. 2007 for details). At the outlet boundary we impose a convective outflow boundary condition, in order to represent the outlet as an open boundary. For the convection velocity we take the free stream velocity, and hence the boundary condition becomes

$$
\left(\partial_{t}+U_{c} \partial_{x}\right) \boldsymbol{U}=0
$$

with $U_{c}=1$. We find that (2.16) performs satisfactorily, and also that the results are fairly insensitive to the exact choice of $U_{c}$, as was reported by Ruith et al. (2003). Finally, we impose the constraint of axisymmetry, and then the equations are advanced forwards in time.

The constraint of axisymmetry is found to be sufficient to ensure convergence, the flow relaxing to a steady state as time is advanced. We find that the resulting steady flow qualitatively resembles, at each $x$ station, the profiles (2.3)-(2.5), even though those are only strictly valid as $x \rightarrow \infty$. However, (2.6)-(2.8) do not hold at finite $x$. Useful measures of the flow can be obtained by fitting it to the profiles (2.3)-(2.5) in order to obtain $\delta_{\mathrm{loc}}, \alpha_{\mathrm{loc}}, \kappa_{\mathrm{loc}}$, approximate local values of the vortex core radius, axial velocity defect and swirl parameter, respectively. We find that $\delta_{\mathrm{loc}}$ slowly increases with $x$, while $\alpha_{\text {loc }}$ decreases and $\kappa_{\text {loc }}$ remains approximately constant, which is qualitatively compatible with (2.6)-(2.8). The effect of the viscous spreading thus sees a gradual increase in the local swirl strength $q$ given by (2.9), which implies a gradually more stable local flow (see e.g. Mayer \& Powell 1992), as expected.

\subsection{Solving the stability problem}

For the stability problem we must numerically solve the eigenvalue problem (2.12). Rather than formulating (2.12) into a large matrix equation (as done, for example, by Ehrenstein \& Gallaire 2005 and Åkervik et al. 2008), we solve the eigenvalue problem with a time stepping methodology. This methodology uses the fact that, beginning with the Navier-Stokes DNS code, only relatively minor alterations are required to be able to time step the LNS equations (2.10). For the linear perturbations satisfying (2.10) we use Dirichlet boundary conditions on the inlet and lateral boundaries, and at the outlet we use the convected boundary condition (2.16).

The process of integrating the linearized equations forwards by a time $t_{A}$ defines a new linear operator $\mathbf{C}$ which, in terms of the eigenvalue problem (2.12), can be symbolically written as

The eigenvalue problem,

$$
\mathbf{C} \equiv \exp \left(-\boldsymbol{B}^{-1} \boldsymbol{A} t_{A}\right)
$$

$$
c q=\lambda q,
$$

is solved for this new operator using the implicitly restarted Arnoldi method. For this we use the 'znaupd()' routine in the ARPACK software library, see Lehoucq, Sorensen $\&$ Yang (1998) for full details. As it proceeds the Arnoldi method continually supplies vectors $\boldsymbol{q}$ for which the calling program must compute $\boldsymbol{C} \boldsymbol{q}$ and return the result. This is achieved by time stepping the linearized equations, and thus none of the operators $\boldsymbol{A}, \boldsymbol{B}$ or $\boldsymbol{C}$ is ever constructed in full. The Arnoldi procedure terminates when the requested number of eigenvalues and eigenvectors have converged to a specified tolerance. In terms of the converged eigenvalues $\lambda$ of (2.18), the frequency eigenvalues $\omega$ are simply given by

$$
\omega=\frac{i \log \lambda}{t_{A}} .
$$

Typically we use $t_{A}=0.25$, corresponding to 50 time steps with $\Delta t=0.005$. 
To validate the computational code and the Arnoldi eigenvalue method we computed results for an artificial parallel base flow, imposing periodic axial boundary conditions. This correctly recovered, as expected, the local stability spectrum. For all calculations in the remainder of the paper we adopt the inlet and outlet boundary conditions described above, which are appropriate for global modes and the global stability spectrum.

\section{Global stability of an artificial parallel vortex}

\subsection{Introduction to the global spectrum}

Our central results on the global stability of a non-parallel Batchelor vortex will be presented in $\S 4$. As a prelude, in this section we shall discuss the global stability of the artificial parallel approximation to the Batchelor vortex. The global stability of an artificial parallel flow is a natural intermediate case between the fully local and global approaches, and is instructive to study. This intermediate analysis differs from a fully local analysis by the introduction of boundary conditions at upstream and downstream locations. This is, therefore, a true global stability analysis and all the features of the global spectra in $\S 4$ will be present here. However, there is the significant advantage that, owing to the parallel flow, the form of the spectrum is described analytically: the global modes are precisely the absolute instability modes. It is intended that this section will provide a useful introduction to global spectra, and also allow discussion of some computational and convergence issues, separately from the detailed results of $\S 4$.

We determine a base flow by following the parallel approximation described after (2.8) with $\alpha=0.9091, \kappa=-1.1000$ and $\delta=1$. Specifically:

$$
U=1-0.9091 \mathrm{e}^{-r^{2}}, \quad V=0, \quad W=\frac{-1.1}{r}\left(1-\mathrm{e}^{-r^{2}}\right) .
$$

The Reynolds number $R e=500$ is chosen for this example.

\subsection{Computation and convergence}

Computation of the global stability spectrum proceeds exactly as described in $\S 2.4$. We use 257 grid points in the axial direction and 127 in the radial direction. For the eigenvalue solver we use an Arnoldi basis of size 50 and seek 25 eigenvalues and eigenvectors, converged to a relative tolerance of $10^{-4}$. The computational domain is denoted by $0 \leqslant x \leqslant x_{\max }$ and $0 \leqslant r \leqslant r_{\max }$. We use $r_{\max }=10$ throughout, however, an important convergence issue surrounds the choice of $x_{\max }$. The purpose of this subsection is to describe, based on empirical observations, how the choice of $x_{\max }$ affects the computed global spectrum.

Empirically, we have found that in our computations the eigenvalue solver cannot fully resolve eigenvectors in which the magnitude of the largest components is more than about $10^{6}$ times that of the smallest components. We suspect that this phenomenon is caused by a combination of the non-normality of the linear operator and issues of numerical precision (rather than, e.g. reflections from the boundaries, Doaré \& de Langre 2006). Now, the global mode eigenvectors $\boldsymbol{q}(x, r)$ have the form (3.4) (see below for justification of the formula), and so display strong exponential growth in the axial direction. These two factors combine to impose an important restriction on $x_{\max }$ :

$$
\left|\operatorname{Im}\left(k_{0}\right)\right| x_{\max } \lesssim \log 10^{6}
$$




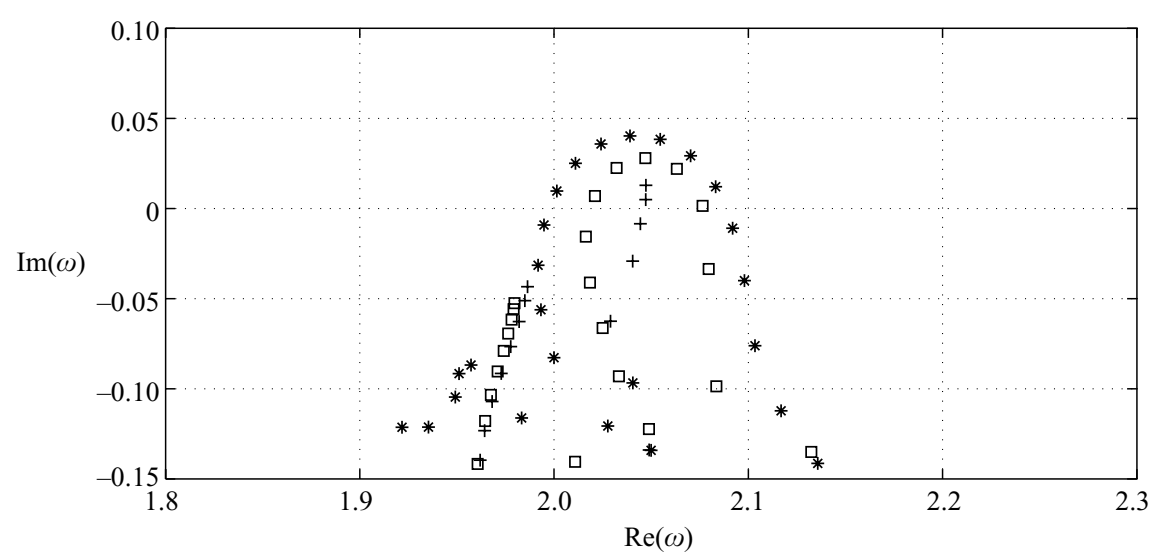

FIGURE 1. Global frequency spectrum for the parallel vortex (3.1) with $m=-2$. Computed eigenvalues are shown for: $x_{\max }=20(+), x_{\max }=40(\square)$ and $x_{\max }=60(*)$.

The specific value of $10^{6}$ encountered in our computations is probably not universal. Varying the number of axial and radial grid points was found to have no effect on the value, but it might be possible to improve upon $10^{6}$ with more numerical precision or better numerical methods in the future.

Figure 1 shows the global mode spectrum for azimuthal wavenumber $m=-2$, computed for various values of $x_{\max }$. The plot demonstrates the typical trend: if $x_{\max }$ is so large that (3.2) is violated then the leading global mode is simply not resolved. In that case the eigenvalues arrange themselves onto a curve which passes above the location of the true global mode (this curve is in fact the $10^{6}$ contour of the resolvent norm of $\mathbf{C}$ ). As $x_{\max }$ decreases, the convective non-normality of the system also decreases, so the resolvent norm of $\mathbf{C}$ tightens about its eigenvalues rendering them less sensitive to perturbations (see $\$ 3.3 .2$ of Schmid \& Henningson 2001). Correspondingly, the hoop of eigenvalues shrinks, until it begins to collapse into a single line, rather like a zip fastener. This first occurs when the leading global mode becomes fully resolved. Further decreasing $x_{\max }$ does not affect the leading mode, indicating that it is converged, but may help the convergence of the higher modes as the zipping process continues and extends the line of converged eigenvalues. In all of our calculations and testing, the appearance of a branch of global modes in which the eigenvalues lie along a hoop shape rather than lying on a line has in this way been an indicator of eigenvalues which are not converged.

For the simple $x$-independent flow being considered, it is permissible to reduce $x_{\max }$ to any desired level. There is no danger of excluding from the computational domain a location where the physics is especially important, as all axial locations are equivalent from a physical point of view. In our calculations, the only lower limit we found on $x_{\max }$ is that there should be sufficient room to represent the shape of the global mode: we find that a value of about twice the axial wavelength is sufficient. This is because it is not possible computationally to achieve exact equivalence of all axial locations, as near to the boundaries the influence of the boundary conditions is felt.

All of these considerations will be equally important in $\S 4$ when we try to obtain converged global modes for the non-parallel base flow, and the experience gained here will prove instructive in steering our computations. However, there is of course one significant difference: different axial locations are not all equivalent in a non-parallel 
base flow, and consequently a more careful justification for the choice of $x_{\max }$ will be required.

\subsection{Global modes and absolute instability}

The global modes for an $x$-independent flow, such as studied in this section, are known to be precisely the absolute modes of the flow. To see this, note that it is possible to take an axial Fourier transform of the stability equations (2.10), even though we do not do so for the global stability problem. The Fourier transform directly leads to the standard derivation of absolute (instability) modes, and their dominance in the limit $t \rightarrow \infty$, with $x$ fixed. The limit of $t \rightarrow \infty$ with $x$ fixed must also give the dominant global mode of (2.12), and hence the two coincide. The absolute modes, and hence the global modes, are therefore given by

$$
\omega_{0}=\omega\left(k_{0}\right) \text { such that }\left.\frac{\mathrm{d} \omega(k)}{\mathrm{d} k}\right|_{k=k_{0}}=0,
$$

where $\omega(k)$ is the dispersion relation for the $x$-independent base flow. The corresponding eigenvector has the form

$$
\boldsymbol{q}(x, r)=\boldsymbol{q}_{k_{0}}(r) \times \mathrm{e}^{\mathrm{i} k_{o} x},
$$

where $\boldsymbol{q}_{k_{0}}(r)$ is the radial profile of the absolute mode. The global mode spectrum therefore comprises a set of points at the absolute frequencies $\omega_{0}$. Additionally, each $\omega_{0}$ acts as a square-root type branch point in the complex frequency plane (see, for example, Huerre \& Rossi 1998). This corresponds to a continuous branch of the spectrum emanating from each $\omega_{0}$.

The equivalence, described above, of global modes and absolute modes in a parallel flow holds exactly in the limit of infinite domain size. However, in a finite domain a discrete spectrum of global modes which satisfy the boundary conditions is expected (Nichols, Chomaz \& Schmid submitted). Thus, in computations the continuous branches of the spectrum will actually be represented by discrete sequences of eigenvalues emanating from each $\omega_{0}$. As for the global frequencies themselves, provided that $x_{\max }$ is chosen to give numerically converged results as empirically described in $\S 3.2$, then the computational domain is large enough to mimic an infinite domain and a good agreement with $\omega_{0}$ is indeed obtained, as our following results now demonstrate.

Figure 2 shows the global spectra of (3.1) for $m=-1$ and $m=-2$, computed with $x_{\max }=40$ and $x_{\max }=20$, respectively. Note that we may restrict to $m \leqslant 0$ without loss of generality: sending $m \rightarrow-m$ simply sends $\omega \rightarrow-\omega^{*}$ and the spectrum is reflected in the imaginary axis. For $m=0$ the flow is very stable and the spectra are not of interest. For $m=-1$ there are three branches of global modes in figure 2(a), each corresponding to an absolute mode. To find the absolute modes we have solved the one-dimensional eigenvalue problem associated with a local stability analysis to find $\omega(k)$ by a standard spectral method (Heaton 2007a). The absolute frequencies and their associated wavenumbers are identified as $\left(\omega_{0}, k_{0}\right)=(0.98+0.031 \mathrm{i}, 0.42-0.18 \mathrm{i})$, $(0.47-0.0075 \mathrm{i}, 1.2-0.042 \mathrm{i})$ and $(0.96-0.023 \mathrm{i}, 0.88-0.14 \mathrm{i})$. In figure $2(a)$ we see that each absolute mode does indeed mark a point at which a sequence of global modes begins, giving a global spectrum of the expected form. The global modes at the head of each branch are found to agree well with the associated absolute mode (3.4). Only small differences arise, primarily near the inlet and outlet, presumably because the boundary conditions do not perfectly model the open nature of these boundaries. On taking a Fourier transform of the global eigenmode at the head of each branch, we find a single distinct peak. These peaks occur at $k=0.41,1.2$ and 

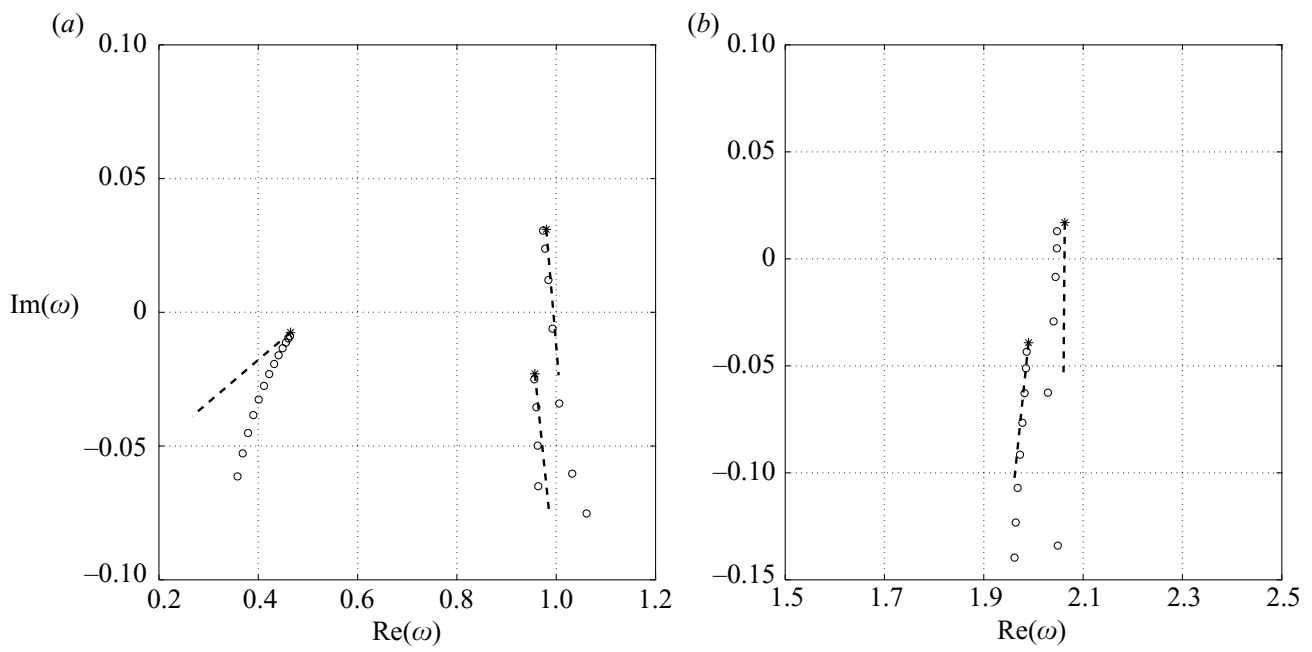

FIGURE 2. The global frequency spectrum for the parallel vortex (3.1): (a) $m=-1,(b) m=-2$. Computed eigenvalues are shown by $\bigcirc$. Absolute frequencies $(*)$ and the dashed lines are shown for comparison, see the text for explanation.

0.92 , respectively, in good agreement with $\operatorname{Re}\left(k_{0}\right)$ from the absolute wavenumbers. We note that within each branch the global modes are qualitatively similar, but with small differences visible in their $x$ dependence and their radial profiles. The differences between branches are more marked, because of the different absolute mode to which each is associated. As a final comparison with theory, Nichols et al. (submitted) predict that the branch cut emanating from each absolute frequency $\omega_{0}$ should do so in the direction given by

$$
\arg \left(\omega-\omega_{0}\right)=\arg \left(\left.\frac{\mathrm{d}^{2} \omega(k)}{\mathrm{d} k^{2}}\right|_{k=k_{0}}\right) .
$$

We have calculated this direction, and it is plotted on figure $2(a)$ by the dashed lines emanating from each absolute frequency. A good agreement is seen between the alignment of the computed global modes and the alignment of the dashed lines, giving further confidence that our computations are functioning correctly.

In figure $2(b)$ a very similar picture is seen for $m=-2$. Now the global modes show correspondence with two absolute modes at $\left(\omega_{0}, k_{0}\right)=(2.1+0.017 \mathrm{i}, 0.99-0.63 \mathrm{i})$ and $(2.0-0.039 \mathrm{i}, 2.1-0.29 \mathrm{i})$. As before, the absolute frequencies and the associated direction (3.5) are shown in on figure 2(b). In this case Fourier transforms of the global modes at the head of each branch give dominant wavenumbers $k=0.98$ and 2.1, again in good agreement with the values of $\operatorname{Re}\left(k_{0}\right)$. Thus, overall a good correspondence is seen between the local absolute modes and the global modes at the head of each branch. The remaining small differences in the eigenvalues, as visible in figure 2, and the eigenmodes, which are not exactly $\propto \mathrm{e}^{\mathrm{i} k_{o} x}$, must be due to limitations in computational accuracy. In particular, one suspects that some error may be due to limitations in how well the boundary conditions model the open nature of the flow.

In summary, the global spectra and the global modes conform to the theory of absolute modes which is available for $x$-independent base flows. Physically, global stability corresponds to fixing a spatial domain of interest within the flow, $0 \leqslant x \leqslant$ $x_{\max }$, and hence of all the possible solutions of (2.10) those with zero group velocity 

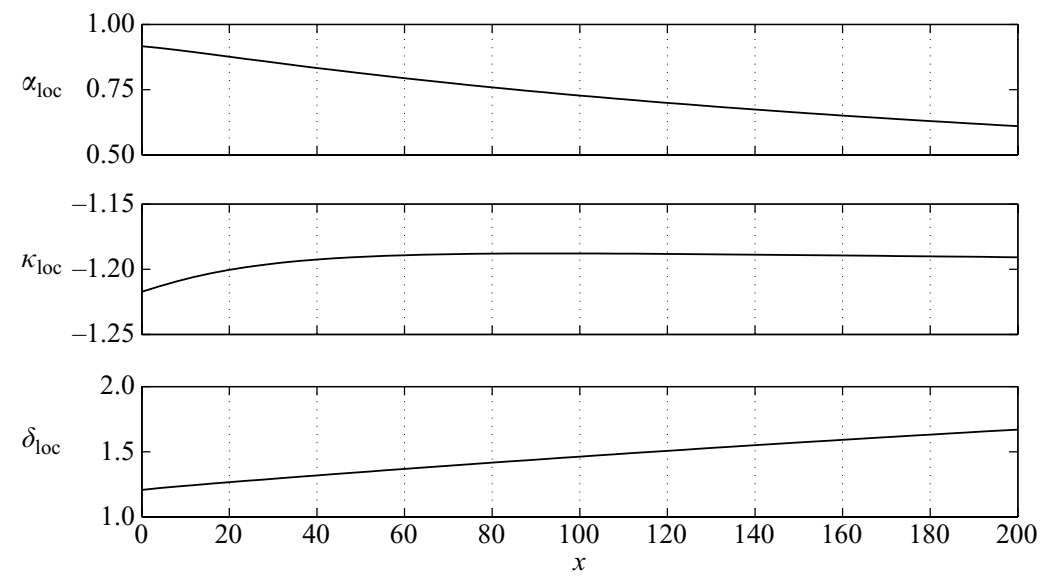

FIGURE 3. Local approximations of the axial velocity defect, swirl parameter and vortex core radius taken from the non-parallel base flow. Values obtained by fitting $U(x, r)$ to $(2.3)$ and $W(x, r)$ to $(2.5)$ at each $x$ station.

(3.3) are the relevant ones. The computations presented give a concrete example of this process at work, and prepare the way for the more complicated problem of non-parallel base flow.

\section{Global stability of the non-parallel vortex}

\subsection{Base flow}

For simplicity, we shall limit the presentation of our results to one particular base flow. The set of possible base flows is a very large parameter space, but we shall not attempt to exhaustively map it out. Instead, we shall concentrate on fully exploring the global spectrum for one illustrative case.

The base flow is obtained as described in $\S 2.3$. We use a long computational box $0 \leqslant x \leqslant 250$, take a Reynolds number of $R e=850$ and define inlet conditions by $\alpha(0)=0.9000, \kappa(0)=-1.1300$ and $\delta(0)=1$. These values are chosen to obtain a vortex which is marginally globally unstable. With axisymmetry imposed, the flow simply relaxes to a steady state as the DNS advances in time. The boundary conditions are designed to model the open nature of the flow but they cannot do so perfectly, especially at the inlet where a profile (with, rather unrealistically, $V=0$ ) is imposed. As a result, the DNS converges to a steady state which noticeably readjusts just downstream of the inlet, and to a lesser extent just upstream of the outlet. Our strategy is to remove these regions containing unphysical effects of the boundary conditions, and to adopt an interior portion of the solution as the base flow for our stability analysis. We stress that the resulting base flow is indeed a steady solution of the Navier-Stokes equations. The problem is simply that a priori we do not know fully suitable inlet profiles to impose, particularly for $V$. We have checked that when restarting the DNS, on the restricted domain and using our base flow to define both initial and inlet conditions, the simulation is immediately in steady state. This confirms that the base flow is a bona fide steady solution of (2.2), and that it is fully consistent to use for stability analysis.

Because the base flow varies only slowly in $x$, it is not very illuminating to plot the components of $\boldsymbol{U}$. Figure 3 shows the local approximations to the axial velocity 

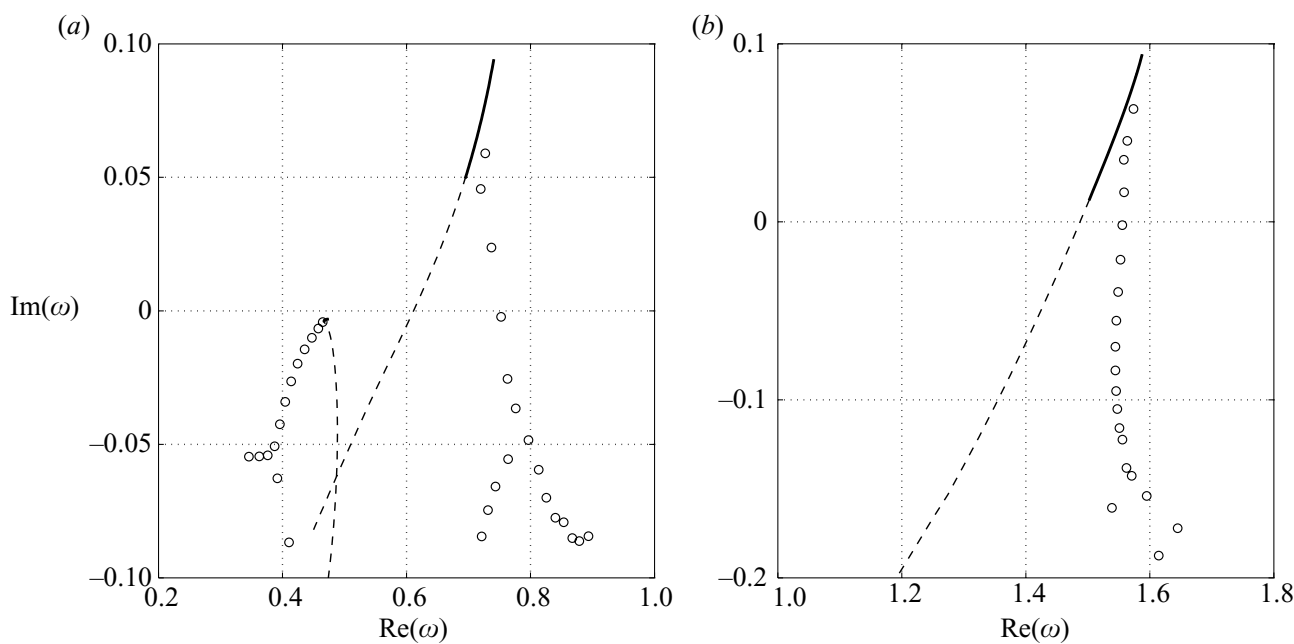

FIGURE 4. The global frequency spectrum for the non-parallel vortex: $(a) m=-1,(b) m=-2$. Computed eigenvalues are shown by $\bigcirc$. The lines show the downstream evolution of local absolute frequencies, as explained in the text.

defect, swirl parameter and vortex core radius, as defined in $\S 2.3$. This plot makes clearer the downstream variation in the $U$ and $W$ components. The radial component $V$ is much smaller throughout, taking a maximum value of $1.3 \times 10^{-3}$ at the inlet and monotonically decaying downstream. As was typical in our calculations, the base flow shows gradual viscous spreading (increase in $\delta_{\text {loc }}$ ) and filling of the wake (decrease in $\alpha_{\text {loc }}$ ). As commented in $\S 2.3$, this means the local properties of the flow become gradually more stable downstream.

\subsection{Global spectrum and global modes}

The global spectrum is computed for the non-parallel base flow in exactly the same manner as described for the parallel base flow in $\S 3$. As in $\S 3$, we have computed the global spectrum using computational domains $0 \leqslant x \leqslant x_{\max }$ for various values of $x_{\max }$, and as expected this parameter has a strong influence on the resulting spectrum. A similar behaviour is obtained in our numerical results to that described above, namely: when $x_{\max }$ is too large the leading global mode in each branch is not fully resolved and the computed eigenvalues lie on a hoop-shaped curve. On reducing $x_{\max }$ the spectrum transforms like a zip fastener and a set of converged eigenvalues lying on a single line is obtained. Global spectra showing converged eigenvalues are plotted in figure 4 using $x_{\max }=25$.

Now, reducing $x_{\max }$ removes part of the base flow from the global stability calculation, and for a spatially developing base flow this requires careful justification. First, our computational experiments lend some empirical support for the validity of the procedure. On reducing $x_{\max }$ the global spectrum transforms in qualitatively the same manner as it did in $\S 3$, when the procedure was certainly valid. Further, the spectra we obtain are numerically converged with respect to further reductions of the $x_{\max }$ parameter. More concretely, we have noted above that the base flow becomes locally more stable further downstream. Particularly, it is the zero-group-velocity disturbances from the local theory that we suspect to impact on the global spectrum. Looking at figure 21 of Olendraru \& Sellier (2002), we see that the zero-group-velocity disturbances indeed become much more stable owing to the downstream decay of 
$\alpha_{\text {loc }}$ seen in figure 3 (N.B. the parameter ' $a$ ' used by Olendraru \& Sellier is equivalent to $-1 / \alpha$ in our notation). This should mean that the most unstable global modes are reliant on the base flow near to the inlet and not affected by the flow further downstream, which is consistent with our findings. Undoubtedly, the regions further downstream must contribute to the true global spectrum of the entire flow, but the relevant eigenvalues must be sufficiently damped that they lie below the resolved regions of the complex $\omega$-plane in figure 4.

Let us now look in detail at the spectra in figure 4 . The spectra are very similar in structure to those shown in figure 2 for the artificial parallel flow, comprising distinct branches of eigenvalues. Each branch has a most unstable mode, and then a sequence of higher modes aligned on a curve underneath the most unstable mode. In figure 4 the point at which each branch of eigenvalues 'splits' is clearly visible. Beyond this point the eigenvectors are not fully resolved and the individual eigenvalues are not converged. Since the base flow is nearly parallel and only varies slowly with $x$, on physical grounds we anticipate that the global spectrum should be intimately related to the zero-group-velocity disturbances of a suitably defined parallel-flow approximation. More importantly, the asymptotic theory of global modes for weakly non-parallel shear flows states that the most unstable global mode is controlled by the local absolute mode (saddle point) with greatest growth rate (Huerre \& Monkewitz 1990). For this reason the local absolute modes have been calculated and are shown by lines on figure 4 .

For each $x$ the local approximation of the flow is formed by discarding $V(x, r)$, and taking the radial profiles of $U(x, r)$ and $W(x, r)$. These are then interpolated onto a Gauss-Lobatto grid in order to perform the spectral calculation for absolute modes $\left(\omega_{0}, k_{0}\right)$. We do indeed find a local absolute mode associated with each of the branches of global modes in figure 4 , and these are shown by the plotted lines. In each case the solid portion of the line shows the locus of $\omega_{0}$ for $0 \leqslant x \leqslant 25$, which is the domain used to compute the global spectrum as described above. The dashed continuation of each line shows the locus of $\omega_{0}$ for $0 \leqslant x \leqslant 200$. As anticipated the local absolute modes become more stable further downstream, in each case $\operatorname{Im}\left(\omega_{0}\right)$ is monotonically decreasing as $x$ increases. A natural consequence is that the global stability of the flow is most strongly dictated by the form of the flow at inlet. Hence, inlet conditions in an experiment, or the choice of inlet location in a numerical computation, are important factors for influencing the global stability.

Asymptotic theory for a nearly parallel base flow (Huerre \& Rossi 1998) predicts the location of the global mode at the head of each branch as follows: to a first approximation, the global mode at the head of each branch is given by the point with greatest imaginary part on the locus of the corresponding local absolute mode. At second order, a correction to the global mode is obtained which has negative imaginary part, implying that the first approximation is actually an upper bound on the growth rate of the global mode at the head of the branch. Figure 4 shows that our results are consistent with both of these predictions. An appealing physical interpretation of the 'upper bound' result is that each global mode might be thought of as a different weighted average of the local properties at each $x$. The maximum in local absolute growth rate occurs only at $x=0$ and therefore is not attained by a global mode. However, as a cautionary note, Nichols et al. (submitted) have shown that generally it may be possible for global modes to exist with larger growth rate than the local absolute mode. This can occur if a sequence of global modes emanates upwards from the mode at the head of the branch instead of emanating downwards, as is seen here and as was also seen in $\S 3$. 

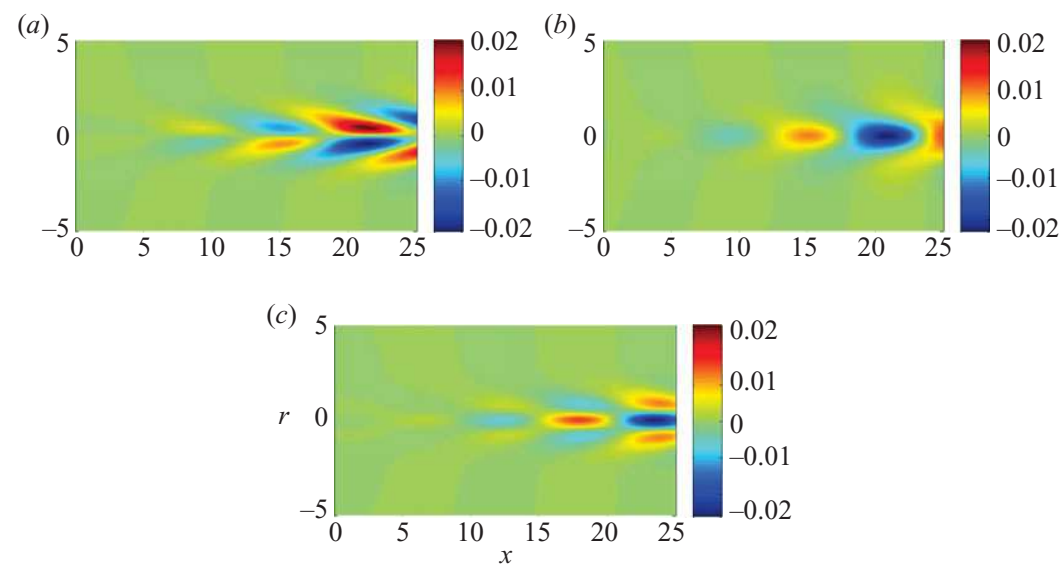

Figure 5. Global mode for $m=-1$ and $\omega=0.727+0.0590$ i. $(a) \operatorname{Re}(u),(b) \operatorname{Re}(v)$ and (c) $\operatorname{Re}(w)$.
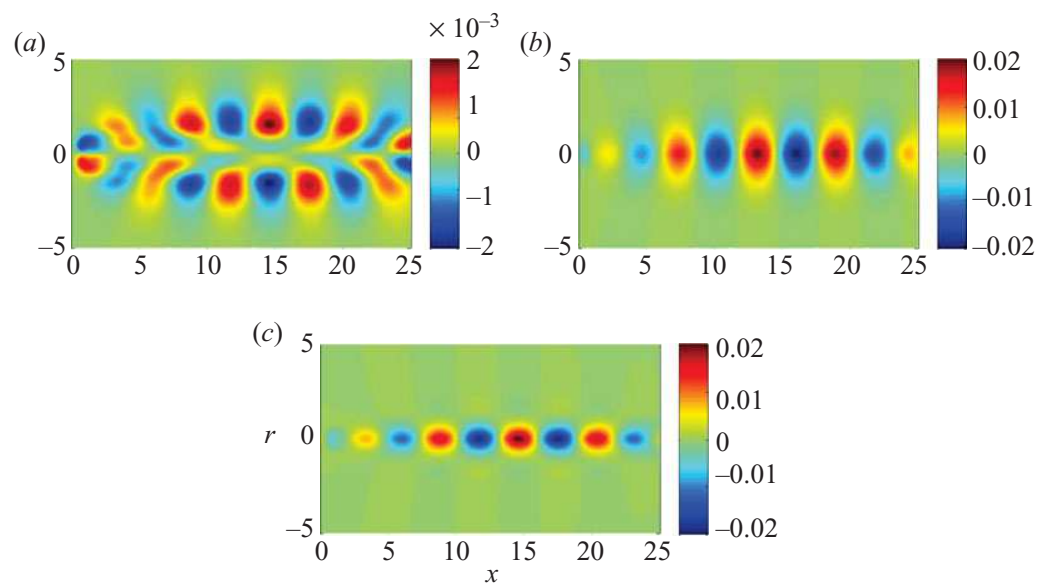

FIgURE 6. Global mode for $m=-1$ and $\omega=0.465+0.00420$ i. $(a) \operatorname{Re}(u),(b) \operatorname{Re}(v)$ and (c) $\operatorname{Re}(w)$.

Figures 5-7 show the velocity fields associated with the global modes at the head of the three distinct branches seen in figure 4. As in $\S 3$, the higher modes within the same branch have qualitatively similar eigenvectors as the mode at the head of the branch, but more marked differences are seen between branches. In each case a good qualitative agreement is found between the global mode and the associated local absolute mode but a precise quantitative agreement does not occur, as was the case for the eigenvalues. Partly, this is because a single axial location must be chosen to define a local absolute mode for the comparison, and different choices are possible. In any case no single choice is expected to yield a perfect agreement, since the global spectrum combines the properties of all locations into one single measure. The qualitative agreement that we do see is found in the radial profiles, and also in the axial wavelengths. The primary global mode for $m=-1$ shown in figure 5 is estimated to have dominant wavenumber $k=0.49$, found by taking a Fourier transform of the velocity components. If for argument's sake we choose the inlet for the location of comparisons, this compares to $\operatorname{Re}\left(k_{0}\right)=0.40$ for the local absolute mode. For the 

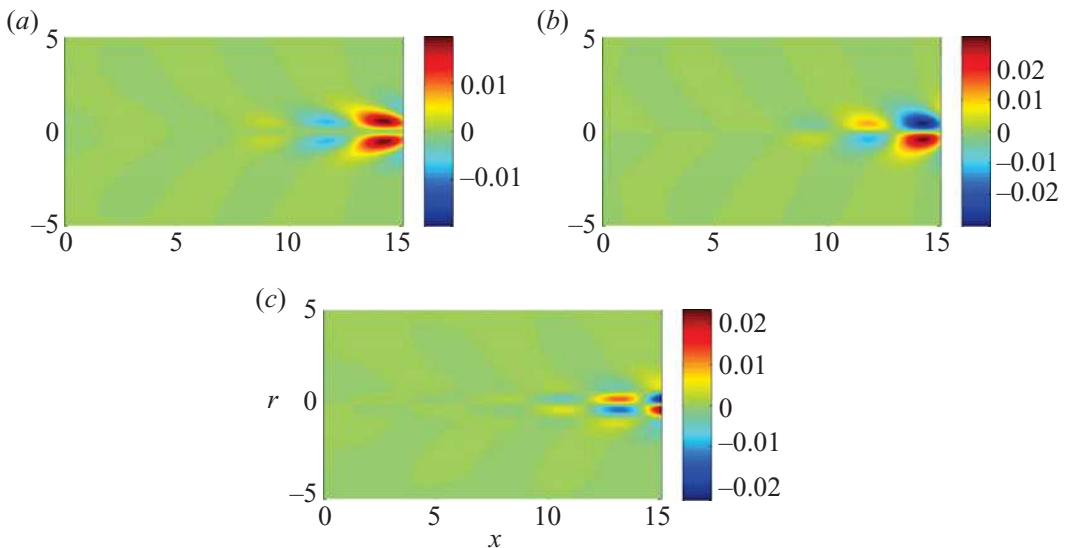

FIGURE 7. Global mode for $m=-2$ and $\omega=1.57+0.0635$ i. $(a) \operatorname{Re}(u),(b) \operatorname{Re}(v)$ and (c) $\operatorname{Re}(w)$.

$m=-1$ global mode at the head of the other branch, shown in figure 6 , the velocity components have very different characteristics. In particular the axial wavelength is much shorter, and the axial velocity component is much weaker than the other two components for this mode, in contrast to figure 5. For the global mode in figure 6 we estimate $k=1.07$ compared to $\operatorname{Re}\left(k_{0}\right)=1.02$ for the local absolute mode. For the primary global mode with $m=-2$, shown in figure 7, we estimate $k=1.19$ whereas the local absolute mode has $\operatorname{Re}\left(k_{0}\right)=0.95$ at inlet. In this case, the global mode looks rather different to figures 5 and 6 , because of the conditions on the symmetry axis $r=0$, which require that $u=v=w=0$ for $|m|>1$.

The global spectrum of the non-parallel flow has been discussed in detail for $m=-1,-2$, but what are the implications for the dynamics of the flow? The flow is globally unstable, the most amplified mode being $\omega=1.57+0.0635 i$ for $m=-2$ (for $m \leqslant-3$ we find that all global modes are stable). This indicates that the flow, at least in the linear regime, could behave as a resonator, with the entire flow beating at this frequency. However, since the global instability has a modest growth rate it may take some time for the global mode to grow sufficiently to dominate the dynamics. To understand the short-time dynamics we must consider superpositions of the global modes, and this is the subject of the next section.

\subsection{Superposition of global modes}

The global spectrum is a basis for the solutions of the LNS equations (2.10), and so should encapsulate the behaviour of all possible solutions. All solutions to (2.10) must tend to the most unstable global mode in the limit $t \rightarrow \infty$, but at finite times one must take into account the entire spectrum and not simply the most unstable mode. This entails a study of non-modal stability (Schmid 2007), which mathematically is in strong analogy to the non-modal stability theory in parallel flows (for solutions to the Orr-Sommerfeld equation). The calculation method is unchanged, but the eigenvectors in our case are two-dimensional global modes and have a different physical interpretation.

The method of analysis is fully reviewed by Schmid \& Henningson (2001), so we shall only define the quantities we shall use. The kinetic energy of a solution to (2.10) 

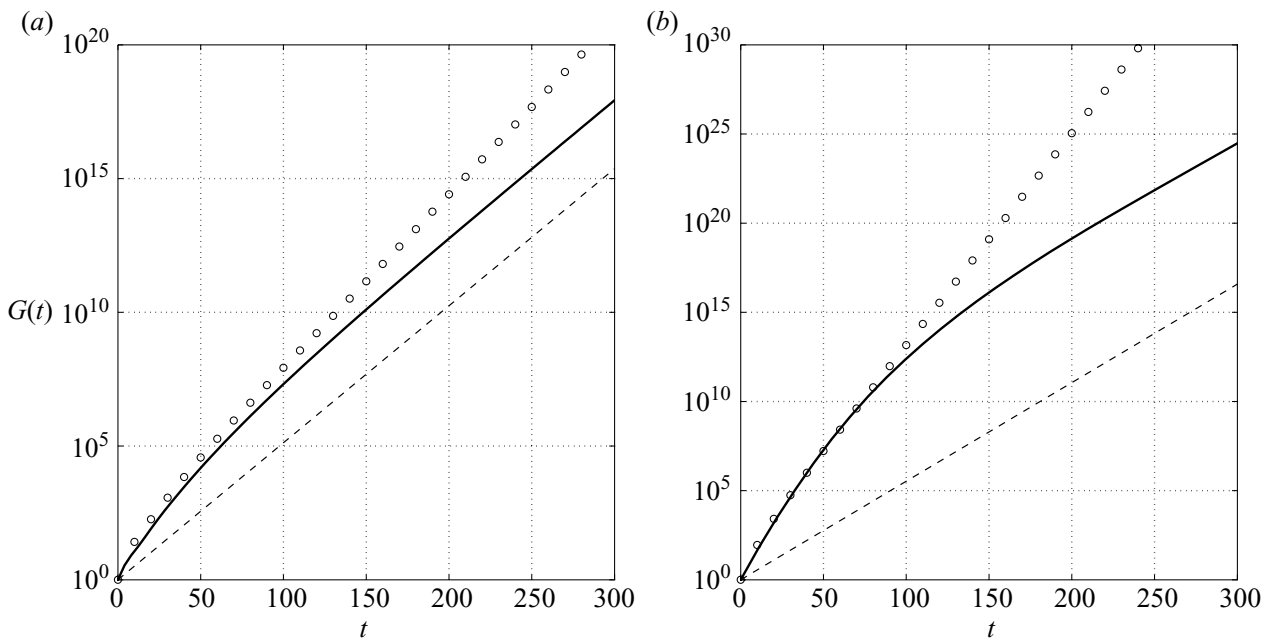

FIGURE 8. Transient growth gain curves (solid lines) for (a) $m=-1$ and $(b) m=-2$. For comparison, dashed lines show the growth rate of the primary global mode and $\bigcirc$ symbols show $\widetilde{G}(t)$, a measure of convective growth.

is given by

$$
E(t)=\frac{1}{2} \int_{0}^{x_{\max }} \int_{0}^{r_{\max }}\left(|u|^{2}+|v|^{2}+|w|^{2}\right) r=\mathrm{d} r \mathrm{~d} x,
$$

and the quantity $E(t) / E(0)$ is referred to as the energy amplification. Non-modal stability is quantified in terms of the maximum possible energy amplification, maximized over all initial conditions:

$$
G(t)=\max _{\boldsymbol{u}(0)} \frac{E(t)}{E(0)} .
$$

The quantity $G(t)$ is known as the gain.

The gain curves $G(t)$ for $m=-1,-2$ have been calculated from the global spectra and are shown in figure 8 by the solid lines. In each case a dashed line shows the growth rate of the primary global mode, which is expected to dictate the growth at long times as discussed above. It is evident that as $t \rightarrow \infty$ the gain curves become parallel to the dashed lines, and the primary global mode does indeed control the large- $t$ growth. However, at shorter times the gain curve increases much more rapidly, most strikingly for $m=-2$ (figure $8 b$ ). While the global modes are intimately related to absolute instability, the rapid short-time growth is in contrast related to convective instability. This statement is justified by considering the example of a convectively unstable wave packet which grows as it convects downstream. The energy $E(t)$ of such a wave-packet solution increases at first, but eventually the wave packet must leave the computational domain and its energy is lost, so the energy growth seen is inherently transient in nature. This is termed 'convective non-normality' by Chomaz (2005) and is a typical growth mechanism, owing to the fact that in any parallel flow there are convective disturbances which grow at a faster rate than the absolute mode. This mechanism contrasts with 'lift-up non-normality', the mechanism which leads to transient growth in local analyses (Chomaz 2005).

In order to estimate the strength of the convective non-normality we need to study convective instabilities, and hence a local parallel-flow approximation is required. As 
(a)

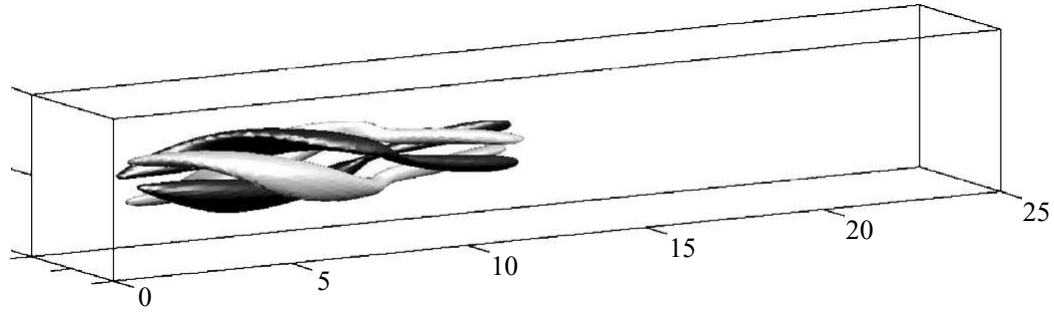

(b)
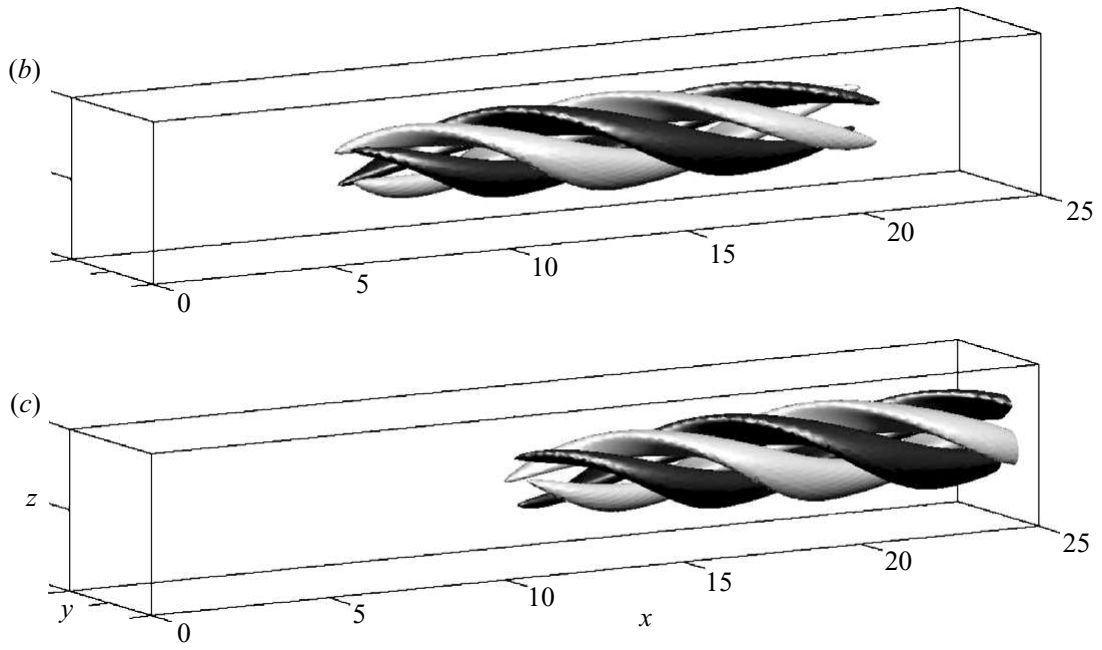

FIgURE 9. Perspective views of the $t=40$ optimal perturbation at $(a) t=0,(b) t=20$ and (c) $t=40$. The visualisation shows \pm isosurfaces of $u$.

we did in $\S 4.2$ for comparing the global modes to local absolute modes, we shall use the base flow at the inlet for the comparison. A measure of the strength of convective instabilities is obtained by calculating the gain for disturbances of axial wavenumber $k$ to the parallel flow obtained from the base flow profiles at inlet. We denote this gain by $\widetilde{G}(k, t)$, and it is calculated using the method employed by Heaton $(2007 a)$. This is then maximized over all wavenumbers to obtain a measure of the maximum convective transient growth:

$$
\widetilde{G}(t)=\max _{k \in \mathbb{R}} \widetilde{G}(k, t) .
$$

Since the base flow varies slowly in $x, \widetilde{G}(t)$ provides a reasonable estimate of the maximum convective growth possible throughout the domain, and hence $\widetilde{G}(t)$ is an appropriate measure of the maximum growth of convective solutions (such as wave packets). In figure 8 the quantity $\widetilde{G}(t)$ is shown by the circles. It is seen that the short-time rapid growth in the gain curve matches this measure of the maximum convective growth quite well. A perfect agreement is not expected because of the approximation of the entire base flow by its inlet profiles in the definition of $\widetilde{G}(t)$, but good agreement is seen nevertheless. We conclude that $\widetilde{G}(t)$ provides a good estimate of the global gain curve at short times.

Herein, let us concentrate on $m=-2$, for which the more striking transient growth is seen. Confirmation that the rapid growth seen at short times in figure $8(b)$ is convective in nature is given by figure 9 , which shows visualisations of the disturbance that attains 
(a)

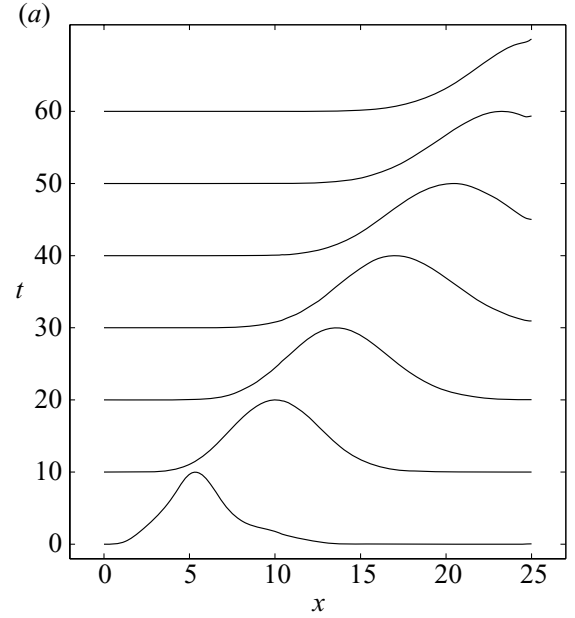

(b)

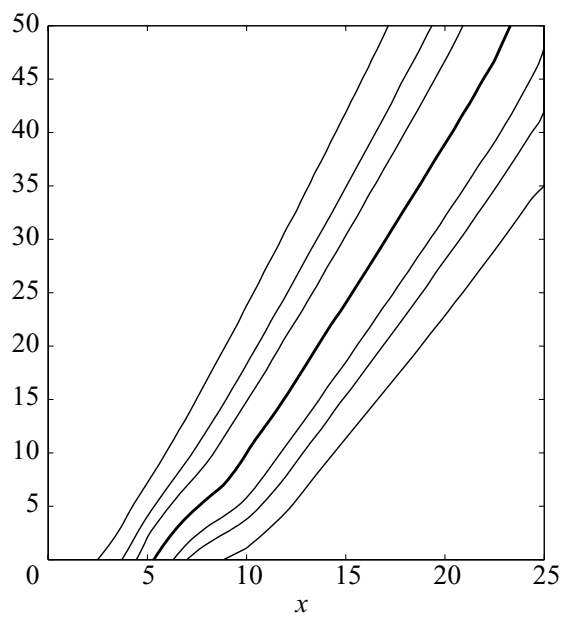

FIGURE 10. (a) Waterfall plot of the scaled energy density of the $t=40$ optimal, plotted for $t=0,10, \ldots, 60$. $(b)$ The axial location of greatest energy density for the same disturbance (thick line). The thin lines show the locations of $50 \%, 75 \%$ and $90 \%$ of maximum energy density.

the maximal growth at $t=40$. Initially the disturbance is localized near to the inlet with peak amplitude at $x \simeq 5$. At later times the disturbance grows significantly and also convects downstream, having the appearance of a localized wave packet. The convection downstream is additionally evident in figure 10(a), which shows the radially integrated kinetic energy as a function of $x$. In figure 10(b) the location of the maximum of radially integrated kinetic energy is used to show the location of the wave packet as a function of time. After a short initial transient, the wave packet convects downstream at a uniform speed of about 0.3 . Lines showing $50 \%, 75 \%$ and $90 \%$ of maximum energy are also shown, and these indicate that the wave packet is also spreading as it propagates downstream. This is reminiscent of the growth and spreading of convective instabilities triggered by a point force (Huerre \& Rossi 1998), although here the initial disturbance (figure $9 a$ ) is much less strongly localized.

As a further exploration of the short-time convective stage of the transient growth gain curve, figure 11(a) shows the gain curves obtained when $x_{\max }$, the length of the computational domain, is varied. The dashed line shows the growth rate of the primary global mode, and the circles show convective growth measure $\widetilde{G}(t)$, exactly the same as in figure $8(b)$. The gain curves show a dependence on $x_{\max }$, with the greatest gain being found for the largest $x_{\max }$. This is because the rapid convective growth mechanism can be sustained for longer times in a longer computational domain. For smaller $x_{\max }$ the convective wave packet leaves the domain sooner, meaning the transition between the small- $t$ regime (the circle symbols) and the large- $t$ regime (the dashed line) occurs sooner. This process is clearly seen in figure 11(a), and gives further credibility to the use of $\widetilde{G}(t)$ as an approximate measure of the convective growth.

Finally, we note that all the transient growth results presented in this section are based upon linear algebra manipulations of the global mode spectrum. We do not possess the complete global mode spectrum, and typically we seek a maximum of 50 eigenvalues and eigenvectors from the Arnoldi process described in $§ 2.4$. For the transient growth calculations this constitutes a severe restriction of the full DNS 
(a)

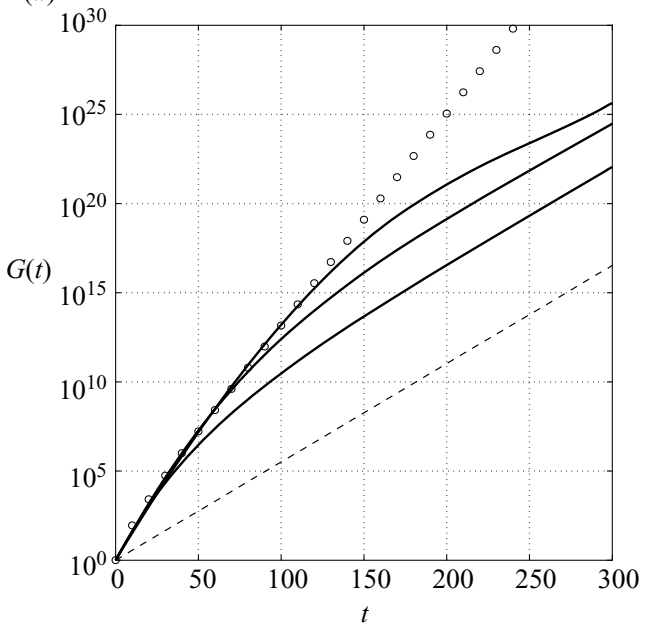

(b)

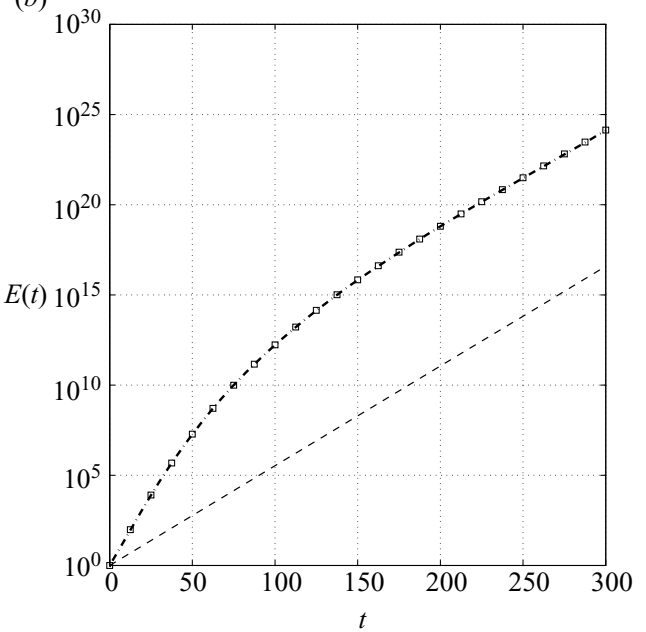

FiguRE 11. (a) Transient growth gain curves (solid lines) for $m=-2$, comparing $x_{\max }=15$, 25 and 60. Dashed lines and circles as in figure 8. $(b)$ comparison of $E(t)$ for the $t=40$ optimal using the global spectrum (dash-dot line) and DNS of the optimal initial condition ( $\square$ symbols).

solution space onto a vector space of the 50 most unstable modes. From the experience of parallel flows (Schmid \& Henningson 2001) it is expected that the transient growth results will indeed be converged, even when a large number of very stable modes are omitted. To check this explicitly we have performed a linearized DNS of (2.10) using the $t=40$ optimal initial condition, as calculated from from the global spectrum and visualised in figure $9(a)$, as initial condition for the DNS. Figure 11(b) shows the comparison between the evolution of the energy $E(t)$ in the DNS and the energy obtained solely from the computed global mode spectrum. The two agree very well, and a similar level of agreement is also found for the detailed shape of the disturbance as visualised in figure 9 . This gives a posteriori support for the validity of the approach we have used, and confirms that the transient growth we have obtained is genuine, despite the reduction of the full dynamics onto only 50 of the most unstable global modes.

To summarise, we find that large levels of non-modal growth are possible via appropriate superpositions of the global modes. At short times rapid growth occurs through a convective mechanism, over and above the growth associated with the primary global mode. The quantity $\widetilde{G}(t)$ is found to give a good indication of the energy gain in this regime at small times. $\widetilde{G}(t)$ is computed from a local analysis, and so this is a computationally cheap estimate of the convective transient growth. The energy gain at large times is controlled by the primary global mode, but again a computationally cheap estimate could be made, from absolute growth rates in a local analysis. For the flow considered here the convective growth at short times is very strong when compared to the global growth of the primary mode, and it is unlikely that the large- $t$ regime of the primary global mode will be accessible. This indicates that the flow, being only marginally globally unstable, is more likely to display amplifier dynamics than resonator dynamics in practice.

We have restricted our investigation to one illustrative, marginally unstable, flow. In other cases the balance between amplifier and resonator dynamics could be different, 
e.g. for a strongly globally unstable flow one might expect the global modes and resonator dynamics to be dominant, with a relatively small amplifier effect seen only at small times.

\section{Conclusions}

In this paper we have investigated the global stability of the non-parallel Batchelor vortex. This family of swirling wakes and jets has a number of applications, as well as serving as an interesting prototype for global stability analyses of nearly parallel base flows. With such analyses still in their infancy, we have fully discussed the numerical methods used and convergence issues encountered. For detailed results we have restricted to one particular illustrative case of a swirling-wake base flow, and in presenting results we have paid particular attention to correspondence of the global mode spectrum with existing asymptotic theory.

In $\S 3$ the basic theory and concepts of global stability and global modes were reviewed in the context of an artificial parallel base flow. This was a convenient opportunity to discuss in full our computational methods, and also is a convenient intermediate case between local and global stability concepts. We found that $\operatorname{Im}\left(k_{0}\right)$ plays an important role in determining the maximum domain size which will yield converged eigenvalues on a finite-precision computer. As $x_{\max }$ is decreased, more global modes become resolvable, the global spectrum transforming like a zip fastener. The computed spectra (figure 2) reveal clearly the structure anticipated theoretically: global modes at the absolute frequencies and a branch cut emanating down from each. To our knowledge this is the first direct observation of this structure in a global stability analysis of the Navier-Stokes equations.

In $\S 4$ we gave our main results on the global stability of the non-parallel base flow. The base flow, computed via DNS as an exact steady solution of NavierStokes, was very close to parallel (figure 3) and only marginally globally unstable. The global spectra have a similar structure to the artificial parallel base flow, and the correspondence of global modes with absolute modes of a local approximation to the base flow was explored. Although precise agreement is not expected, a good level of agreement was established. In $\S 4.3$ non-modal stability was investigated, and superpositions of global modes were found representing convective instability and amplifier dynamics at short times. At longer times the global modes and resonator dynamics prevail.

In the wider context of global stability of nearly parallel flow, we believe that the level of correspondence in $\S 4.2$ between the global modes and local absolute modes has not previously been demonstrated. The only other studies which show a global spectrum of a nearly parallel base flow are those of Ehrenstein \& Gallaire (2005) and Åkervik et al. (2008) for Blasius boundary-layer flow, and in these cases no correspondence with local absolute modes is given. The spectra presented by Ehrenstein \& Gallaire (2005) and Åkervik et al. (2008) have a rather different structure to those in figure 4, superficially resembling the hoop-shaped spectra in figure 1 which arise when $x_{\max }$ is too large. We tentatively suggest that a possible explanation for the lack of a correspondence to a local absolute mode could be due to the value of $x_{\max }$ being too large in these cases. In support of this, we note that figure 4(a) of Åkervik et al. (2008) shows a correspondence between their global modes and the local modes with $k \in \mathbb{R}$, rather than the local modes with zero group velocity, as we have found. If a relation such as (3.2) holds in their computations as it did in our own, then on a domain of length $x_{\max }=800$ this would severely restrict the 
range of $\operatorname{Im}(k)$ being resolved, and might explain why correspondence is seen with the local modes with $k \in \mathbb{R}$.

However, it is important to note that much of the dynamics of the LNS equations is only represented by superposition of many global modes. Even if not fully converged owing to a large value of $x_{\max }$, the computed modes are eigenvalues to within the working precision of the computer and may be useful for this purpose. For example, the gain $G(t)$ may actually be fully converged in some restricted domain of small $t$, as it was in the studies of Ehrenstein \& Gallaire (2005) and Åkervik et al. (2008). However extreme care is needed, and in particular the long-time behaviour can never be correct unless the imaginary parts of the leading computed mode and the true global mode agree. If the imaginary part of the leading computed mode does not match the true global mode, then the long-time gain $G(t)$ must necessarily display an incorrect decay rate (in globally stable flows such as the flat-plate boundary layer) or growth rate (in globally unstable flows).

This work was carried out while C. J. Heaton visited LadHyX under a David Crighton Fellowship. The warm welcome extended at LadHyX and the financial support of the David Crighton Fellowship are both gratefully acknowledged.

\section{REFERENCES}

ABID, M. 2008 Nonlinear mode selection in a model of trailing line vortices. J. Fluid Mech. $\mathbf{6 0 5}$, $19-45$.

Abid, M. \& BRachet, M. E. 1998 Direct numerical simulations of the Batchelor trailing vortex by a spectral method. Phys. Fluids 10, 469-475.

Åkervik, E., Ehrenstein, U., Gallaire, F. \& Henningson, D. S. 2008 Global two-dimensional stability measures of the flat plate boundary-layer flow. Eur. J. Mech. B/Fluids 27, 501-513.

Åkervik, E., Hoepffner, J., Ehrenstein, U. \& Henningson, D. S. 2007 Optimal growth, model reduction and control in a separated boundary-layer flow using global eigenmodes. J. Fluid Mech. 579, 305-314.

Batchelor, G. K. 1964 Axial flow in trailing line vortices. J. Fluid Mech. 20, 645-658.

Blackburn, H. M., Barkley, D. \& Sherwin, S. J. 2008 Convective instability and transient growth in flow over a backward-facing step. J. Fluid Mech. 603, 271-304.

Broadhurst, M. S. 2006 Vortex stability and breakdown: direct numerical simulation and stability analysis using biglobal and parabolised formulations. $\mathrm{PhD}$ thesis, Imperial College, London.

Chomaz, J. M. 2005 Global instabilities in spatially developing flows: non-normality and nonlinearity. Annu. Rev. Fluid Mech. 37, 357-392.

Constantinescu, G. S. \& Lele, S. K. 2002 A highly accurate technique for the treatment of flow equations at the polar axis in cylindrical coordinates using series expansions. J. Comput. Phys. 183, 165-186.

Cossu, C. \& Chomaz, J. M. 1997 Global measures of local convective instabilities. Phys. Rev. Lett. 78, 4387-4390.

Delbende, I., Chomaz, J. M. \& Huerre, P. 1998 Absolute/convective instabilities in the Batchelor vortex: a numerical study of the linear impulse response. J. Fluid Mech. 355, 229-254.

DoArÉ, O. \& DE LANGRE, E. 2006 The role of boundary conditions in the instability of onedimensional systems. Eur. J. Mech. B/Fluids 25, 948-959.

Ehrenstein, U. \& Gallaire, F. 2005 On two-dimensional temporal modes in spatially evolving open flows: the flat-plate boundary layer. J. Fluid Mech. 536, 209-218.

FABRE, D. \& JACQUIN, L. 2004 Viscous instabilities in trailing vortices at large swirl number. J. Fluid Mech. 500, 239-262.

Griffith, M. D., Leweke, T., Thompson, M. C. \& Hourigan, K. 2008 Steady inlet flow in stenotic geometries: convective and absolute instabilities. J. Fluid Mech. 616, 111-133.

Heaton, C. J. $2007 a$ Centre modes in inviscid swirling flows and their application to the stability of the Batchelor vortex. J. Fluid Mech. 576, 325-348. 
Heaton, C. J. 2007b Optimal growth of the Batchelor vortex viscous modes. J. Fluid Mech. 592, 495-505.

Heaton, C. J. \& Peake, N. 2007 Transient growth in vortices with axial flow. J. Fluid Mech. 587, 271-301.

Herbert, T. 1997 Parabolized stability equations. Annu. Rev. Fluid Mech. 29, 245-283.

Huerre, P. 2000 Open shear flow instabilities. In Perspectives in Fluid Dynamics: A Collective Introduction to Current Research (ed. G. K. Batchelor, H. K. Moffatt \& M. G. Worster), pp. 159-230. Cambridge University Press.

Huerre, P. \& Monkewitz, P. A. 1990 Local and global instabilities in spatially developing flows. Annu. Rev. Fluid Mech. 22, 473-537.

Huerre, P. \& Rossi, M. 1998 Hydrodynamic instabilities in open flows. In Hydrodynamics and Nonlinear Instabilities (ed. C. Godreche \& P. Manneville), pp. 81-294. Cambridge University Press.

Khorrami, M. R. 1991 On the viscous modes of instability of a trailing line vortex. J. Fluid Mech. 225, 197-212.

LE Dizès, S. \& FABRE, D. 2007 Large-Reynolds-number asymptotic analysis of viscous centre modes in vortices. J. Fluid Mech. 585, 153-180.

LehoucQ, R. B., Sorensen, D. C. \& Yang, C. 1998 ARPACK Users' Guide: Solution of Large-Scale Eigenvalue Problems with Implicitly Restarted Arnoldi Methods. SIAM.

Leibovich, S. 1978 The structure of vortex breakdown. Annu. Rev. Fluid Mech. 10, 221-246.

Leibovich, S. \& Stewartson, K. 1983 A sufficient condition for the instability of columnar vortices. J. Fluid Mech. 126, 335-356.

Lessen, M., Singh, P. J. \& Paillet, F. 1974 The stability of a trailing line vortex. Part 1. Inviscid theory. J. Fluid Mech. 63, 753-763.

Marquet, O., Lombardi, M., Chomaz, J. M., Sipp, D. \& Jacquin, L. 2009 Direct and adjoint global modes of a recirculation bubble: lift-up and convective non-normalities. J. Fluid Mech. 622, $1-21$.

Marquet, O., Sipp, D., Chomaz, J. M. \& Jacquin, L. 2008 Amplifier and resonator dynamics of a low-Reynolds-number recirculation bubble in a global framework. J. Fluid Mech. 605, $429-443$.

Mayer, E. W. \& Powell, K. G. 1992 Viscous and inviscid instabilities of a trailing vortex. J. Fluid Mech. 245, 91-114.

Nichols, J. W. 2005 Simulation and stability analysis of jet diffusion flames. PhD thesis, University of Washington.

Nichols, J. W., Chomaz, J. M. \& Schmid, P. J. 2009 Twisted absolute instability in lifted flames. Phys. Fluids 21, 015110.

Nichols, J. W. \& Schmid, P. J. 2008 The effect of a lifted flame on the stability of round fuel jets. J. Fluid Mech. 609, 275-284.

Nichols, J. W., Schmid, P. J. \& Riley, J. J. 2007 Self-sustained oscillations in variable-density round jets. J. Fluid Mech. 582, 341-376.

Olendraru, C. \& Sellier, A. 2002 Viscous effects in the absolute-convective instability of the Batchelor vortex. J. Fluid Mech. 459, 371-396.

Olendraru, C., Sellier, A., Rossi, M. \& Huerre, P. 1999 Inviscid instability of the Batchelor vortex: absolute-convective transition and spatial branches. Phys. Fluids 11, 1805-1820.

PIER, B. 2002 On the frequency selection of finite-amplitude vortex shedding in the cylinder wake. J. Fluid Mech. 458, 407-417.

Pier, B. 2008 Local and global instabilities in the wake of a sphere. J. Fluid Mech. 603, 39-61.

Ruith, M. R., Chen, P., Meiburg, E. \& Maxworthy, T. 2003 Three-dimensional vortex breakdown in swirling jets and wakes: direct numerical simulation. J. Fluid Mech. 486, 331-378.

Schmid, P. J. 2007 Nonmodal stability theory. Annu. Rev. Fluid Mech. 39, 129-162.

Schmid, P. J. \& Henningson, D. S. 2001 Stability and Transition in Shear Flows. Springer.

Stewartson, K. \& Brown, S. N. 1985 Near-neutral centre-modes as inviscid perturbations to a trailing line vortex. J. Fluid Mech. 156, 387-399.

YIN, X., Sun, D. \& WEI, M. 2000 Absolute and convective instability character of slender vortices. Phys. Fluids 12, 1062-1072. 